\title{
High Expression of MDM2 and the p53 Protein is Predictive Biomarkers for Poor Prognosis of Oesophageal Squamous Cell Carcinoma
}

This article was published in the following Dove Press journal: Cancer Management and Research

Juan $Y \mathrm{e}^{1,2, *}$

Lin Zhang ${ }^{2-4, *}$

Zhongwen $\mathrm{Li}^{1}$

Runduan $\operatorname{Lin}^{3}$

Yiling Song ${ }^{3}$

Huanhe $\mathrm{Ni}^{2}$

Xiaoxia Gou (1D ${ }^{\prime}$

Rongzhang $\mathrm{Xie}^{4}$

'Department of Head and Neck Oncology, The Second Affiliated Hospital of Zunyi Medical University, Zunyi, Guizhou, People's Republic of China; ${ }^{2}$ Department of Experimental Research, Sun Yat-Sen University Cancer Center, State Key Laboratory of Oncology in South China, Collaborative Innovation Center for Cancer Medicine, Guangzhou, Guangdong, People's Republic of China; ${ }^{3}$ Department of Clinical Laboratory, Sun Yat-Sen University Cancer Center, State Key Laboratory of Oncology in South China, Collaborative Innovation Center for Cancer Medicine, Guangzhou, Guangdong, People's Republic of China; ${ }^{4}$ Department of Clinical Laboratory, Yunfu People's Hospital, Yunfu, Guangdong, People's Republic of China

*These authors contributed equally to this work
Background and Objective: In the present study, we detected the expression of MDM2 and p53 in oesophageal squamous cell carcinoma (OSCC) specimens, studied their relationship with the survival of OSCC patients, and explored the potential of MDM2 and p53 to serve as predictive OSCC tumour markers.

Patients and Methods: Through immunohistochemistry and fluorescence in situ hybridization (FISH), we detected the expression of MDM2 and the p53 protein in 157 OSCC specimens that met the inclusion and exclusion criteria. After scoring the results, Pearson's chi-square test and Cox regression were used for analysis.

Results: The results showed that the rates of high MDM2 and p53 expression in OSCC tissues were $60.5 \%$ and $51.0 \%$, respectively. The expression levels of MDM2 and p53 in OSCC were significantly positively correlated $(p<0.001, \mathrm{r}=0.414)$. In addition, the pathological metastasis (M) status and MDM2 protein expression in OSCC were significantly correlated $(p=0.027)$, and high expression of the $\mathrm{p} 53$ protein was positively correlated with OSCC transfer $(p=0.005)$, pathological node status $(p=0.008)$, and clinical stage $(p=0.003)$. Kaplan-Meier survival analysis showed that the high expression of MDM2 and p53 was significantly related to the poor prognosis of OSCC. Moreover, subgroup analysis of the TNM staging of OSCC patients showed that the high expression of MDM2 and p53 was significantly correlated with poor OS and DFS of OSCC patients in either stage I-II or III-IV patients. Both univariate and Cox multivariate analyses showed that p53 and MDM2 can be used as independent factors for the prognosis of OSCC patients. Finally, our FISH detection results for MDM2 showed that the high expression of MDM2 was significantly correlated with the amplification of MDM2 $(p=0.015)$.

Conclusion: This study shows that MDM2 and p53 can be used as independent predictors of the prognosis of patients with oesophageal squamous cell carcinoma.

Keywords: oesophageal squamous cell carcinoma, biomarkers, MDM2, p53

\section{Introduction}

Oesophageal cancer is a common tumour type in the digestive tract and the fourth leading cause of cancer-related death in the world. ${ }^{1-4}$ As the most common histopathological type of oesophageal cancer, OSCC accounts for $95 \%$ of oesophageal cancers. ${ }^{3,5-7}$ OSCC usually has a poor prognosis, and its five-year survival rate is less than $20 \% .^{8-10}$ Therefore, the study of potential tumour biomarkers in OSCC is of great significance for the treatment and diagnosis of OSCC.
Correspondence: Rongzhang Xie

Tel +867668861761

Fax +867668822491

Email xierz2046@163.com

Xiaoxia Gou

$\mathrm{Tel} / \mathrm{Fax}+8685127596472$

Email angela7695@163.com 
p53 is an important tumour suppressor protein. Multiple in-depth studies have been conducted since the gene was first reported in $1979 .{ }^{11-13}$ It was originally thought that the p53 gene was an oncogene, but with deepening research in the past decade, the function of p53 as a tumour suppressor gene was gradually revealed. Mutations of the p53 gene have been found in more than $50 \%$ of human tumour tissues. ${ }^{14}$ This is the most common genetic change in tumours, indicating that changes in this gene are likely to be the main cause of human tumours. After the p53 gene mutates, due to the change in its spatial conformation, it loses its regulatory effect on cell growth, apoptosis and DNA repair. ${ }^{15}$ The mutated p53 gene is transformed from a tumour suppressor gene into an oncogene. The p53 gene has also been found to have the highest correlation with human tumours. ${ }^{16}$ Therefore, p53-related gene therapy has important application prospects for cancer patients.

Researchers discovered that MDM2 is expressed in various tissues and can suppress the expression of $\mathrm{p} 53$ by regulating the negative feedback loop. ${ }^{17}$ Therefore, after blocking the interaction between MDM2 and p53, it is possible to effectively treat human tumours that retain wild-type $\mathrm{p} 53$ by reactivating the $\mathrm{p} 53$ tumour suppressor function. ${ }^{18,19}$ MDM2 expression has been found in gastric cancer, colorectal cancer, liver cancer, ovarian cancer and pancreatic cancer. ${ }^{20-25}$ The overexpression of MDM2 may affect the occurrence and proliferation of tumours by inhibiting the function of $\mathrm{p} 53$. In some cases, researchers detected MDM2 expression without a p53 mutation, indicating that it can replace the inactivated p53 mutation. ${ }^{26}$

In this study, we used immunohistochemical staining to evaluate the expression of MDM2 and $\mathrm{p} 53$ protein in OSCC specimens and studied the relationship between the expression of MDM2 and the p53 and prognosis in OSCC. Therefore, our research aims to establish a prognostic model of OSCC patients based on the expression of MDM2 and the $\mathrm{p} 53$ protein and explore its value in clinical practice. We present the following article in accordance with the STROBE reporting checklist.

\section{Methods}

\section{Patients and Clinical Samples}

In this study, clinical paraffin specimens of 157 patients with OSCC were collected and sectioned, and the clinical data of these 157 OSCC patients were combined for retrospective analysis. All OSCC patients who participated in this retrospective analysis underwent surgery at the Cancer Center of
Sun Yat-sen University, Guangzhou, China, from November 2000 to February 2002. The patients with M1 in our study were treated with salvage surgery to improve symptoms of severe esophageal obstruction and were not resected. Patients who met the following four criteria were included in our study: i) the pathological types of all patients were confirmed by pathologists in the pathology department and were not diagnosed with other tumours; ii) no antitumour therapy had been performed previously; iii) the patients were not accompanied by other serious diseases of other systems; iv) the patients' clinical and follow-up data were well documented. The patients' exclusion criteria were as follows: i) the patients' clinical statistics and data were incomplete; ii) the patients had severe digestive dysfunction; iii) the patient also had other types of malignant tumours. All patients in this study provided scientific research-related informed consent and completed follow-up records by telephone or outpatient follow-up after surgery. This study was submitted to the Research Ethics Committee of Sun Yat-sen University Cancer Center for review and approval (China) (GZR2015093). The trial was conducted in accordance with the Declaration of Helsinki.

\section{Immunohistochemistry}

Pathological sections ( $4 \mu \mathrm{m}$ thick) were placed in a $65^{\circ} \mathrm{C}$ oven for 30 minutes and then deparaffinized with a gradient of alcohol and xylene. They were then placed in a citrate solution $(10 \mathrm{mmol} / \mathrm{L}, \mathrm{pH} 6.0)$, and the microwave method was used for antigen retrieval. Endogenous peroxidase was blocked with $0.3 \% \mathrm{H}_{2} \mathrm{O}_{2}$ for 15 minutes at room temperature. The specimen was incubated with antihuman MDM2 mouse (1:200, ZSGB-BIO, Cat. No. ZM0425) and anti- p53 mouse (1:200, ZSGB-BIO, Cat. No. ZM-0408) antibodies overnight $\left(4^{\circ} \mathrm{C}\right)$. The secondary antibody (1:1000, Beyotime, Cat. No. A0208) was incubated for 30 minutes at room temperature. 3,3'Diaminobenzidine tetrahydrochloride (DAB) was used for visualization. Finally, the pathological sections were counterstained with haematoxylin, dehydrated, washed in xylene, and sealed with neutral glue for optical microscopy.

Two pathologists independently evaluated all pathological sections by the following two parameters: (a) score of positive area of pathological section $(0,<5 \% ; 1,6 \%$ $25 \% ; 2,26-50 \% ; 3,51-75 \% ; 4,>75 \%$ ) and (b) positive intensity score $(0=$ no signal, $1=$ weak, $2=$ medium, $3=$ strong). The final score was calculated by the following formula score $=a \times b$. 


\section{Fluorescence in situ Hybridization $\left(\mathrm{FISH}^{27}\right)$ Analysis}

Pathological sections $(4 \mu \mathrm{m})$ were placed in a $50^{\circ} \mathrm{C}$ incubator for 2 to 3 hours. The slide specimen was removed and immersed in a denaturation solution of $70 \%$ formamide $/ 2 \times \mathrm{SSC}$ at $70 \sim 75^{\circ} \mathrm{C}$ for $2 \sim 3$ minutes. Next, $10 \mathrm{~mL}$ of the denatured or pre-annealed DNA probe was dropped onto the denatured and dehydrated slide specimen, which was placed in a humidified dark box at $37^{\circ} \mathrm{C}$ overnight (approximately $15-17$ hours). The hybridized slide specimens were placed in a preheated $42 \sim 50^{\circ} \mathrm{C}$ volume fraction of $50 \%$ formamide $/ 2 \times \mathrm{SSC}$ and washed 3 times for 5 minutes each. Then, $150 \mathrm{~mL}$ of blocking solution was added to the hybridization site of the slide and incubated at $37^{\circ} \mathrm{C}$ for 20 minutes. Then, $150 \mathrm{~mL}$ of avidin-FITC was added to the specimen and incubated at $37^{\circ} \mathrm{C}$ for 40 minutes. The specimen was removed, placed in the preheated $42 \sim 50^{\circ} \mathrm{C}$ eluent and washed 3 times for 5 minutes each. Next, $150 \mathrm{~mL}$ of blocking solution II was added to the hybridization part of the slide specimen, which was covered with plastic wrap and incubated at $37^{\circ} \mathrm{C}$ for 20 minutes. The slide was removed and allowed to dry naturally. A $200 \mathrm{~mL} \mathrm{PI/antifade} \mathrm{dye} \mathrm{solution} \mathrm{was} \mathrm{dropped} \mathrm{onto}$ the slide specimen. DAPI was used to counterstain the sections. FISH slides were evaluated with a fluorescence microscope (DM1245; Leica, Germany) using appropriate filter sets. Orange (centromeric region) and green (target gene) signals were counted in 60 non-overlapping tumour cell nuclei from three tumour areas. Dual colour FISH probes were obtained from zytoVision (Bremerhaven, Germany).

\section{Statistical Analysis}

All statistical analyses were performed using SPSS version 22.0 (SPSS Institute, IL, USA). According to the median immunohistochemical score, we divided the patients into a high expression group and a low expression group. Pearson's chi-square test and Fisher's chi-square test were used to analyse the relationship between different expression levels of MDM2 and p53 and pathological parameters of oesophageal squamous cell carcinoma. Kaplan-Meier survival analysis was used to examine the relationship between MDM2 and p53 expression levels and patient prognosis. A Cox proportional hazards model was used to evaluate factors related to prognosis through single factor and multivariate analyses. Statistical significance was set at $p<0.05$.

\section{Results General Characteristics}

In this study, the median age of the enrolled patients was 62 years (range: $35-90) ; 125$ patients were male $(79.6 \%)$ and 32 patients were female (20.4\%). Overall, 48 (30.6\%), 69 (43.9\%) and $40(25.5 \%)$ patients were diagnosed with histological grade III, III disease, respectively. According to TNM and WHO classification criteria, there were 33 (21\%) and 124 (79\%) patients with T1-2 and T3-4 disease, 84 (53.5\%) and 73 (46.5\%) patients with N0 and N1-3 disease, 135 (86.0\%) and $22(14.0 \%)$ patients with M0 and M1 disease, and 83 (52.9\%) and 74 (47.1\%) patients with clinical stage I-II and III-IV disease, respectively. ${ }^{19}$ In total,115 (73.2\%) of the enrolled patients died during the follow-up period (Table 1).

Table I Clinical Characteristics of Patients with Esophageal Squamous Cell Carcinoma

\begin{tabular}{|l|c|}
\hline Characteristics & No. of Patients (\%) \\
\hline Total case & 157 \\
\hline Age (Years) & \\
Median, Range & $62,35-90$ \\
$\leq 62$ & $84(53.5 \%)$ \\
$>62$ & $73(46.5 \%)$ \\
\hline Gender & \\
Male & $125(79.6 \%)$ \\
Female & $32(20.4 \%)$ \\
\hline Histological grade & \\
GI & $48(30.6 \%)$ \\
G2 & $69(43.9 \%)$ \\
G3 & $40(25.5 \%)$ \\
\hline Pathological Tumor (T) Status & \\
TI-2 & $33(21.0 \%)$ \\
T3-4 & $124(79.0 \%)$ \\
\hline Pathological Node (N) Status & \\
N0 & $115(73.2 \%)$ \\
NI-3 & $84(53.5 \%)$ \\
\hline Pathological metastasis (M) Status & $73(46.5 \%)$ \\
M0 & \\
MI & $74(47.1 \%)$ \\
\hline Clinical stage & \\
I-II & \\
III-IV & $(86.0 \%)$ \\
\hline Death & \\
No & \\
Yes & \\
\hline
\end{tabular}

Abbreviations: T, tumor; N, node; M, metastasis; TNM, tumor-node-metastasis. 
The Expression Levels of MDM2 and p53 in Oesophageal Squamous Cell Carcinoma and Their Relationship with Clinical Parameters

Using immunohistochemical staining, we detected the expression of MDM2 and p53 in the tumour cell nucleus.
Compared with the negative control (Figure 1E and F), the number of patients in the high MDM2 and high-p53 expression groups was 95 (60.5\%, Figure 1A and B) and 80 (51.0\%, Figure 1C and D), respectively. High expression of p53 was positively correlated with $\mathrm{N}$ status
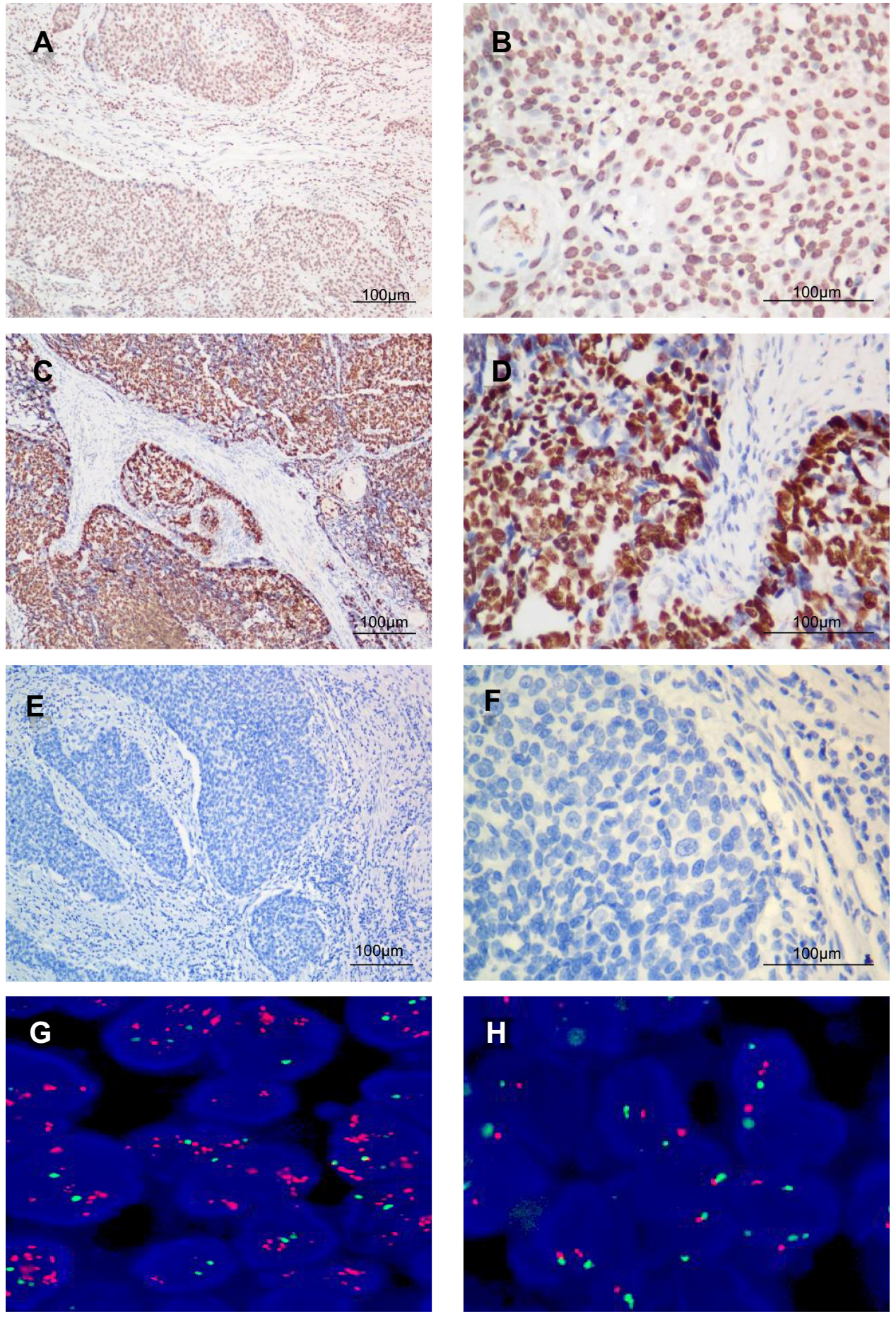

Figure I Immunohistochemical staining for MDM2 and p53 in human oesophageal carcinoma. The data show expression levels of MDM2 (A $\times 100, \mathbf{B} \times 400)$ and $\mathrm{p} 53(\mathbf{C} \times 100$, $\mathbf{D} \times 400)$ compared with the negative control $(\mathbf{E} \times 100, \mathbf{F} \times 400)$ in tumour tissues from patients with OSCC. Amplification of the MDM2 gene $(\mathbf{G} \times 1000)$ compared with the negative control $(\mathbf{H} \times 1000)$ according to FISH in tumour tissues from patients with OSCC. 
$(p=0.005)$ and clinical stage $(p=0.003)$, and high expression of MDM2 $(p=0.027)$ and p53 $(p=0.008)$ was positively correlated with the $\mathrm{M}$ status of oesophageal cancer (Table 2).

\section{The Influence of the Expression Levels of MDM2 and p53 on Patient Prognosis}

The median survival times of OS and DFS of the 157 enrolled patients were 26 months and 23 months, respectively. The cumulative OS rate and DFS rate of the patients in this study were 29 and 31\%, respectively (data not shown). KaplanMeier survival analysis showed that DFS and OS of OSCC patients were positively correlated with the expression levels of MDM2 and p53 $(p<0.05)$ (Figure 2). Univariate analysis showed that gender (DFS, $p<0.003$; OS, $p<0.001$ ), $\mathrm{N}$ status (DFS, $p<0.001$; OS, $p<0.001$ ), M status (DFS, $p=0.005$; OS, $p<0.011$ ), clinical stage (DFS, $p<0.001$; OS, $p<0.001$ ), MDM2 expression (DFS, $p<0.001$; OS, $p<0.001$ ) and $\mathrm{p} 53$ expression (DFS, $p<0.001$; OS, $p<0.001$ ) were closely related to the reduction in patient DFS and OS (Table 3). Multivariate Cox analysis further confirmed that gender (DFS, $p=0.019$; OS, $p=0.005$ ), clinical stage (DFS, $p=0.013$; OS, $p=0.024$ ), MDM2 expression (DFS, $p=0.015$; OS, $p=0.005$ ) and $\mathrm{p} 53$ expression (DFS, $p=0.004$; OS, $p=0.001$ ) were independent predictors of DFS and OS (Table 4).

\section{The Expression Levels and Prognosis of MDM2 and P53 in OSCC Stage I-II and III-IV Patients}

Kaplan-Meier survival analysis showed that there was a statistically significant difference between DFS, OS and MDM2 and p53 expression levels in patients with stage I-II $(83,52.9 \%)$ and stage III-IV (74, 47.1\%) oesophageal cancer (Figure $3, p<0.05$ ). The univariate analysis demonstrated that the high expression levels of MDM2 (DFS, $p=0.003$; OS, $p=0.001$ ) or $\mathrm{p} 53$ (DFS, $p=0.005$; OS, $p=0.001$ ) and MDM2 (DFS, $p=0.010$; OS, $p=0.002$ ) or p53 (DFS,

Table 2 Association of the Expression of MDM2, p53 and Clinicopathologic Parameters in I57 Patients with Esophageal Squamous Cell Carcinoma

\begin{tabular}{|c|c|c|c|c|c|c|c|}
\hline \multirow[t]{2}{*}{ Clinicopathologic Parameters } & \multirow[t]{2}{*}{ Case } & \multicolumn{3}{|c|}{ MDM2 Expression (\%) } & \multicolumn{3}{|c|}{ p53 Expression (\%) } \\
\hline & & Low & High & $p$ value & Low & High & $p$ value \\
\hline \multicolumn{8}{|l|}{ Age(Years) } \\
\hline$\leq 62$ & 84 & $35(4 I .7)$ & $49(58.3)$ & 0.550 & $44(52.4)$ & $40(47.6)$ & 0.370 \\
\hline$>62$ & 73 & $27(37.0)$ & $46(63.0)$ & & $33(45.2)$ & $40(54.8)$ & \\
\hline \multicolumn{8}{|l|}{ Gender } \\
\hline Male & 125 & $45(36.0)$ & $80(64.0)$ & 0.077 & $59(47.2)$ & $66(52.8)$ & 0.361 \\
\hline Female & 32 & $17(53.1)$ & $15(46.9)$ & & $18(56.3)$ & 14 (48.7) & \\
\hline \multicolumn{8}{|l|}{ Histological grade } \\
\hline Grade I & 48 & $19(39.6)$ & $29(60.4)$ & 0.996 & $25(52.1)$ & $23(47.9)$ & 0.880 \\
\hline Grade 2 & 69 & $27(39.1)$ & $42(60.9)$ & & $33(47.8)$ & $36(52.2)$ & \\
\hline Grade 3 & 40 & $16(40.0)$ & $24(60.0)$ & & $19(47.5)$ & $21(52.5)$ & \\
\hline \multicolumn{8}{|l|}{ Pathological Tumor (T) Status } \\
\hline $\mathrm{TI}-2$ & 33 & II (33.3) & $22(66.7)$ & 0.416 & $14(42.4)$ & $19(57.6)$ & 0.392 \\
\hline T3-4 & 124 & $5 I(4 I . I)$ & $73(58.9)$ & & $63(50.8)$ & $61(49.2)$ & \\
\hline \multicolumn{8}{|l|}{ Pathological Node (N) Status } \\
\hline No & 84 & $39(46.4)$ & $45(53.6)$ & 0.056 & $50(64.9)$ & $34(42.5)$ & 0.005 \\
\hline $\mathrm{NI}-3$ & 73 & $23(31.5)$ & $50(68.5)$ & & $27(35.1)$ & $46(57.5)$ & \\
\hline \multicolumn{8}{|l|}{ Pathological metastasis (M) Status } \\
\hline Mo & 135 & $58(43.0)$ & $77(57.0)$ & 0.027 & $72(53.3)$ & $63(46.7)$ & 0.008 \\
\hline MI & 22 & $4(18.2)$ & $18(81.8)$ & & $5(22.7)$ & $17(77.3)$ & \\
\hline \multicolumn{8}{|l|}{ Clinical stage } \\
\hline $\mathrm{I}-\mathrm{II}$ & 83 & $38(45.8)$ & $45(54.2)$ & 0.088 & $50(64.9)$ & $33(41.3)$ & 0.003 \\
\hline III-IV & 74 & $24(32.4)$ & $50(67.6)$ & & $27(35.1)$ & $47(58.8)$ & \\
\hline
\end{tabular}

Notes: Pearson's $X^{2}$ test and Fisher's $X^{2}$ test were used in Table 2. TNM denotes tumor-node-metastasis; bold indicate statistically significant $(p<0.05)$. 

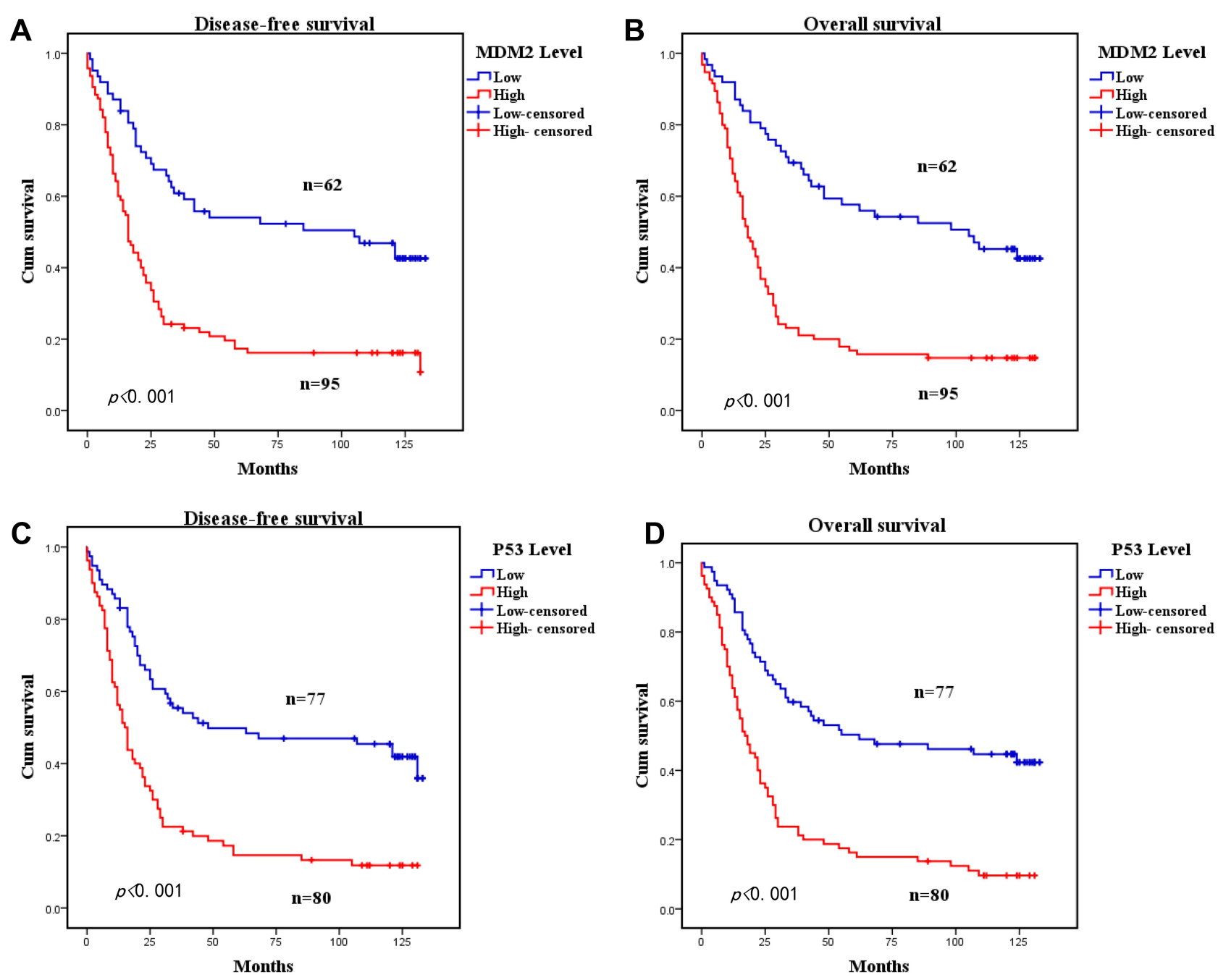

Figure 2 Kaplan-Meier survival analysis in 157 patients with OSCC. (A) Disease-free survival (DFS) and (B) overall survival (OS) curves for patients according the MDM2 protein expression level; (C) DFS and (D) OS curves for patients according to the p53 protein expression level.

$p=0.004$; OS, $p=0.003$ ) in tumour cells were significantly correlated with DFS and OS in stage I-II and stage III-IV patients with OSCC, as shown in Table 3. The expression of MDM2 (DFS, $p=0.040$; OS, $p=0.032$ ) in tumour cells in stage I-II was an independent predictor of DFS and OS according to the multivariate Cox analysis in Table 4. The expression of MDM2 (OS, $p=0.038)$ in tumour cells in stage III-IV was an independent predictor of OS, but there was no association with the expression of p53 in tumour cells in stage I-II and III-IV patients with OSCC.

\section{The Correlation of the Combined} Expression Levels of MDM2 and $\mathrm{p} 53$ in Diverse Cell Populations and Patient Survival In this study, Pearson correlation coefficient and linear regression analyses were used to reveal the relationship between MDM2 and p53. The expression level of MDM2 in oesophageal cancer tissue was positively correlated with the expression of $\mathrm{p} 53(p<0.001, \mathrm{R}=0.414$, Figure $4 \mathrm{~A})$. Compared with patients with high expression of only MDM2 or p53 or low expression of both MDM2 and p53, patients with high expression of both MDM2 and p53 had a worse prognosis, and the DFS and OS times were significantly shortened $(p<0.001, p<0.001$, Figure 4B and C). Multivariate Cox analysis showed that the combined expression of MDM2 and p53 was an independent predictor of DFS and OS (Table 3).

\section{Amplification of MDM2 in OSCC}

High MDM2 expression and MDM2 amplification were detected in 18 tissue samples (18.9\%) (Figure $1 \mathrm{G}$ and $\mathrm{H}$ ). $\mathrm{X}^{2}$ analysis showed that there was a significant 
Table 3 Univariate Cox Regression Analysis for Disease-Free Survival and Overall Survival in Patients with Esophageal Squamous Cell Carcinoma

\begin{tabular}{|c|c|c|c|c|}
\hline \multirow[t]{2}{*}{ Factors } & \multicolumn{2}{|l|}{ Disease-Free Survival } & \multicolumn{2}{|l|}{ Overall Survival } \\
\hline & HR (95\% Cl) & $p$ value* & HR $(95 \% \mathrm{CI})$ & $p$ value* \\
\hline \multicolumn{5}{|c|}{ MDM2 and p53 expression in tumor cells $(n=157)$} \\
\hline Age, years $(\leq 62 />62)$ & $0.790(0.546-1.145)$ & 0.213 & $0.843(0.584-1.217)$ & 0.363 \\
\hline Gender (male/female) & $0.451(0.265-0.768)$ & 0.003 & $0.398(0.231-0.686)$ & 0.001 \\
\hline WHO Grade $(I / 2 / 3)$ & $1.310(1.023-1.676)$ & 0.032 & $1.276(0.999-1.629)$ & 0.051 \\
\hline Tumor $(T)$ status $(I-2 / 3-4)$ & $1.253(0.786-1.997)$ & 0.343 & $1.205(0.757-1.919)$ & 0.432 \\
\hline Nodal $(N)$ status $(0 / 1-3)$ & $2.372(1.628-3.454)$ & $<0.001$ & $2.311(1.591-3.357)$ & $<0.001$ \\
\hline Metastasis $(\mathrm{M})$ status $(0 / \mathrm{I})$ & $1.979(1.228-3.191)$ & 0.005 & $1.885(1.160-3.065)$ & 0.011 \\
\hline Clinical stage (I-II/III-IV) & $2.851(1.943-4.183)$ & $<0.001$ & $2.661(1.823-3.822)$ & 0.001 \\
\hline MDM2 in tumor cells (low/high) & $2.547(1.695-3.827)$ & $<0.001$ & $2.818(1.874-4.238)$ & $<0.001$ \\
\hline p53 in tumor cells (low/high) & $2.566(1.747-3.770)$ & $<0.001$ & $2.805(1.911-4.119)$ & $<0.001$ \\
\hline \multicolumn{5}{|c|}{ MDM2 and p53 expression in stage $I-I I(n=83)$} \\
\hline Age, years $(\leq 62 />62)$ & $0.694(0.393-1.226)$ & 0.209 & $0.808(0.463-1.4 I I)$ & 0.453 \\
\hline Gender (male/female) & $0.435(0.202-0.937)$ & 0.033 & $0.371(0.166-0.826)$ & 0.015 \\
\hline WHO Grade $(1 / 2 / 3)$ & $1.424(0.966-2.098)$ & 0.074 & $1.440(0.984-2.107)$ & 0.061 \\
\hline Tumor $(\mathrm{T})$ status $(I-2 / 3-4)$ & I.I28 (0.6I4-2.073) & 0.699 & $1.089(0.594-1.995)$ & 0.783 \\
\hline Nodal $(N)$ status $(0 / I-3)$ & $1.219(0.517-2.872)$ & 0.651 & $1.167(0.497-2.745)$ & 0.723 \\
\hline Metastasis $(M)$ status $(0 / I)$ & $27.338(2.844-262.790)$ & 0.004 & $41.04(3.718-452.186)$ & 0.002 \\
\hline MDM2 in tumor cells (low/high) & $2.444(\mathrm{I} .35 \mathrm{I}-4.42 \mathrm{I})$ & 0.003 & $2.677(1.484-4.830)$ & 0.001 \\
\hline p53 in tumor cells (low/high) & $2.243(1.272-3.956)$ & 0.005 & $2.600(1.485-4.552)$ & 0.001 \\
\hline \multicolumn{5}{|c|}{ MDM2 and p53 expression in stage III-IV $(n=74)$} \\
\hline Age, years $(\leq 62 />62)$ & $0.894(0.550-1.453)$ & 0.651 & $0.902(0.555-1.466)$ & 0.677 \\
\hline Gender (male/female) & $0.495(0.236-1.039)$ & 0.063 & $0.44 I(0.210-0.927)$ & 0.031 \\
\hline WHO Grade $(1 / 2 / 3)$ & $1.049(0.770-1.428)$ & 0.762 & $1.018(0.747-1.386)$ & 0.911 \\
\hline Tumor $(\mathrm{T})$ status $(\mathrm{I}-2 / 3-4)$ & $0.377(0.166-0.854)$ & 0.019 & $0.429(0.191-0.963)$ & 0.040 \\
\hline Nodal $(N)$ status $(0 / I-3)$ & $1.424(0.648-3.128)$ & 0.379 & $1.597(0.687-3.710)$ & 0.227 \\
\hline Metastasis $(M)$ status $(0 / I)$ & $1.052(0.625-1.773)$ & 0.848 & $1.042(0.615-1.765)$ & 0.878 \\
\hline MDM2 in tumor cells (low/high) & $2.112(1.196-3.730)$ & 0.010 & $2.504(1.407-4.456)$ & 0.002 \\
\hline p53 in tumor cells (low/high) & $2.191(1.285-3.736)$ & 0.004 & $2.278(1.332-3.895)$ & 0.003 \\
\hline
\end{tabular}

Notes: *Univariate analysis, Bold values indicate statistically significant $(p<0.05)$; bold italics values indicate significant differences $(p<0.00 \mathrm{I})$. Abbreviations: $\mathrm{HR}$, hazard ratio; $95 \% \mathrm{Cl}, 95 \%$ confidence interval.

relationship between high MDM2 expression and MDM2 amplification ( $p=0.015$, Table 5$)$. The results showed that high MDM2 expression was significantly correlated with MDM2 amplification.

\section{Discussion}

Various aetiological mechanisms and abnormal apoptosis mechanisms are important causes of tumorigenesis. ${ }^{28}$ Among them, the well-known tumour suppressor gene p53 and its related negative regulator MDM2 play critical roles in the occurrence and progression of cancer. ${ }^{29}$ The expression of MDM2 is upregulated in tumours, which leads to loss of p53 activity by controlling p53 transcriptional activity, protein stability and nuclear localization and causes cell apoptosis and cell cycle disorders. ${ }^{19}$ Studies have shown that $9 \%$ of OSCC patients have increased MDM2 copy numbers, and changes in MDM2 are associated with poor prognosis. ${ }^{30}$ As a common molecular target in the diagnosis and treatment of oesophageal squamous cell carcinoma, the expression of the p53 protein is related to the survival time of patients. ${ }^{31}$ Therefore, $\mathrm{p} 53$ is considered to be an important biomarker to predict the prognosis and survival of OSCC. ${ }^{32}$ MDM2 and p53 play important roles in the clinical diagnosis, treatment and prognosis of oesophageal cancer. ${ }^{33,34}$ In addition, MDM2 and p53 have become two 
Table 4 Multivariate Cox Regression Analysis for Disease-Free Survival and Overall Survival in Patients with Esophageal Squamous Cell Carcinoma

\begin{tabular}{|c|c|c|c|c|}
\hline \multirow[t]{2}{*}{ Factors } & \multicolumn{2}{|l|}{ Disease-Free Survival } & \multicolumn{2}{|l|}{ Overall Survival } \\
\hline & HR (95\% Cl) & $p$ value** & HR (95\% Cl) & $p$ value** \\
\hline \multicolumn{5}{|c|}{ MDM2 and p53 expression in tumor cells $(n=157)$} \\
\hline Gender (male/female) & $0.524(0.305-0.901)$ & 0.019 & $0.465(0.263-0.790)$ & 0.005 \\
\hline WHO Grade $(I / 2 / 3)$ & I.I64 (0.908-I.492) & 0.231 & & \\
\hline Nodal $(\mathrm{N})$ status $(0 / \mathrm{I}-3)$ & I.I48 (0.626-2.105) & 0.656 & $1.231(0.666-2.227)$ & 0.507 \\
\hline Metastasis $(\mathrm{M})$ status $(0 / \mathrm{I})$ & $0.761(0.438-1.321)$ & 0.332 & $0.7 \mid 3(0.409-\mid .24 I)$ & 0.231 \\
\hline Clinical stage (I-II/III-IV) & $2.265(1.184-4.332)$ & 0.013 & $2.124(1.104-4.088)$ & 0.024 \\
\hline MDM2 in tumor cells (low/high) & $1.887(1.226-2.905)$ & 0.015 & $1.923(1.216-3.040)$ & 0.005 \\
\hline p53 in tumor cells (low/high) & $1.321(0.850-2.053)$ & 0.004 & $2.068(1.340-3.194)$ & 0.001 \\
\hline \multicolumn{5}{|c|}{ MDM2 and p53 expression in stage $1-I I(n=83)$} \\
\hline Gender (male/female) & $0.482(0.222-1.046)$ & 0.065 & $0.423(0.188-0.950)$ & 0.037 \\
\hline Metastasis $(\mathrm{M})$ status $(0 / \mathrm{I})$ & 15.150 (I.554-|47.687) & 0.019 & 21.147 (1.897-235.716) & 0.013 \\
\hline MDM2 in tumor cells (low/high) & $1.964(1.030-3.745)$ & 0.040 & $2.023(1.063-3.849)$ & 0.032 \\
\hline p53 in tumor cells (low/high) & $1.585(0.852-2.950)$ & 0.146 & $1.806(0.978-3.336)$ & 0.059 \\
\hline \multicolumn{5}{|c|}{ MDM2 and p53 expression in stage III-IV $(n=74)$} \\
\hline Tumor $(T)$ status $(I-2 / 3-4)$ & $0.491(0.214-1.129)$ & 0.094 & $0.568(0.250-1.289)$ & 0.176 \\
\hline MDM2 in tumor cells (low/high) & $1.621(0.864-3.042)$ & 0.132 & $1.964(1.039-3.711)$ & 0.038 \\
\hline p53 in tumor cells (low/high) & 1.681 (0.924-3.058) & 0.089 & 1.631 (0.893-2.978) & 0.111 \\
\hline
\end{tabular}

Notes: **Cox proportional hazards model, $p<0.05$ considered as statistically significant; bold values indicate statistically significant $(p<0.05)$.

Abbreviations: $\mathrm{HR}$, hazard ratio; $95 \% \mathrm{Cl}, 95 \%$ confidence interval.

important diagnostics of clinical molecular biomarkers for oesophageal cancer. Inhibiting the interaction of MDM2 and p53 may become a new tumour treatment strategy. Currently, some small molecule drugs targeting MDM2p53 have entered the clinical trial stage, and these drugs can restore p53 antitumour activity by blocking the MDM2-p53 interaction in solid and haematologic tumours. $^{29}$

In this study, we found that $\mathrm{p} 53$ expression increased in tumour tissues of OSCC patients. Through the analysis of clinical case data, we found that the expression of p53 was related to the occurrence of oesophageal cancer, with higher p53 expression being correlated with worse differentiation of oesophageal cancer tissue. The survival data of OSCC patients showed that the high expression of p53 was negatively correlated with the survival time of patients and positively correlated with tumour recurrence. In addition, our research found that the expression of MDM2 in OSCC samples was similar to that in gastric cancer, colorectal cancer, liver cancer, ovarian cancer and pancreatic cancer $^{20-23}$ and other diseases. The rate of positive detection of the MDM2 protein in 157 OSCC specimens was significantly higher than that in normal tissues adjacent to cancer.
In this context, we examined the expression patterns of MDM2 and p53 in tumour tissues from 157 patients to determine the predictive value of MDM2 and p53 in different stages of OSCC. We found that the MDM2 protein expression rate in stage I-II OSCC was $61.4 \%$, while that in stage III and IV OSCC was $67.6 \%$, which was similar between the two groups. This suggests that the expression levels of MDM2 and p53 in stage III-IV OSCC are higher than those in stage I-II OSCC and that high expression of MDM2 and p53 may be closely related to increased malignancy and poor prognosis.

Considering the upregulation of MDM2 protein expression in OSCC, we used FISH to determine the amplification of MDM2. Studies have shown that DNA amplification may be one of the reasons for the high expression of MDM2. However, some patients showed upregulation of the MDM2 protein without DNA amplification, indicating that the expression of MDM2 can be regulated by gene amplification as well as by molecular mechanisms, such as transcriptional regulation and posttranscriptional events.

This study has several limitations. We did not test for p53 mutations in the samples. The immunohistochemical 
A

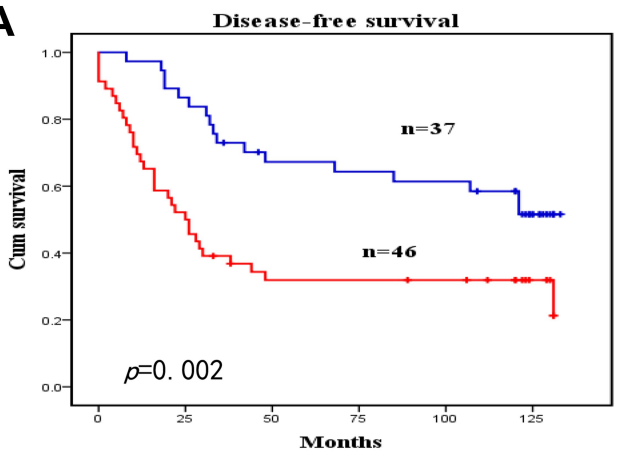

C

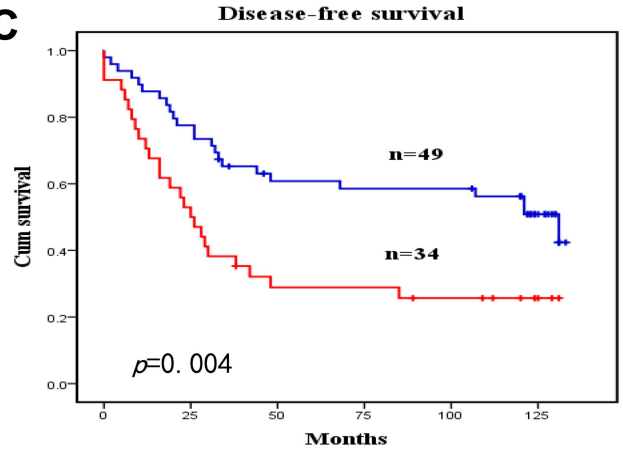

E

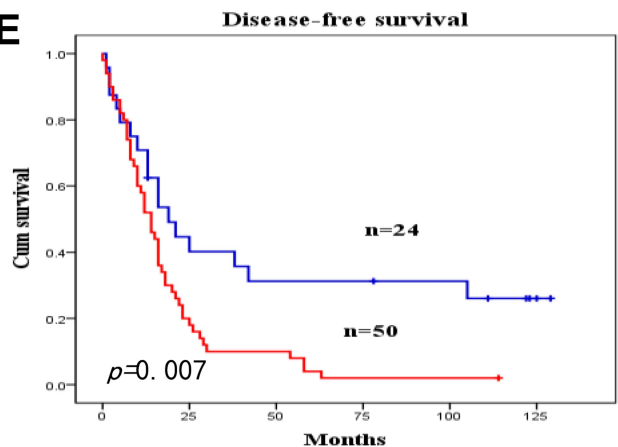

G

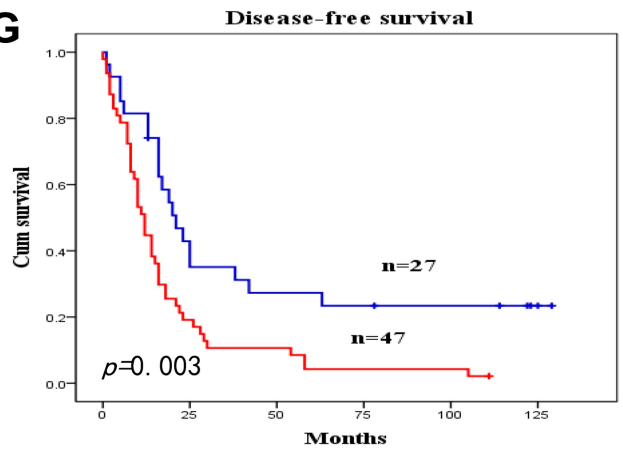

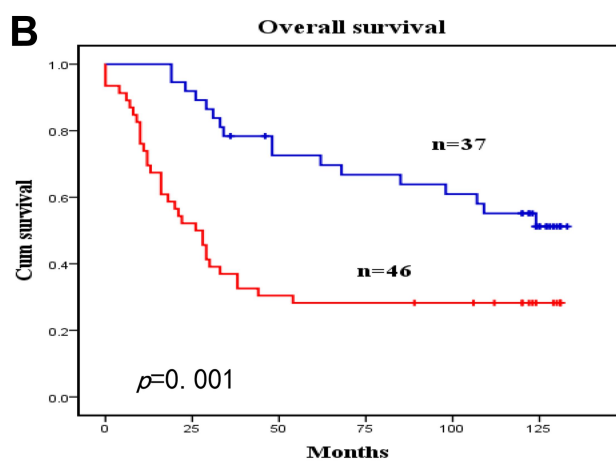

MDM2 Level Glow †Low-censored
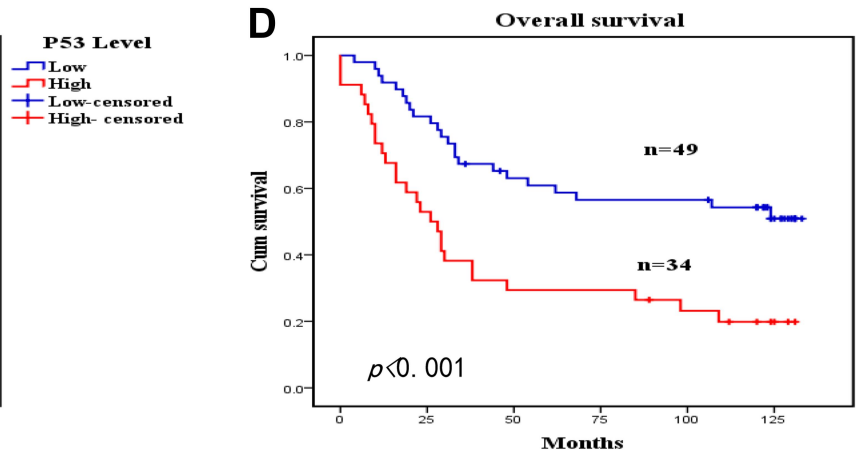

P53 Level ThLow 干-Low-censored MDM2 Level Rllow †Low-censored
+High- censored

$F \quad$ Overall survival

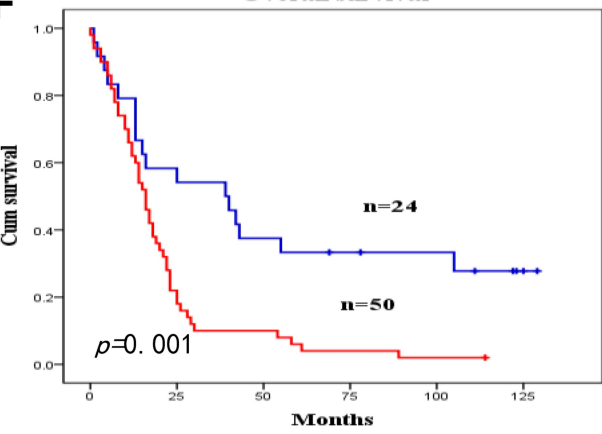

MDM2 Level AnLow 十-Low-censored

H

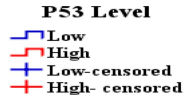

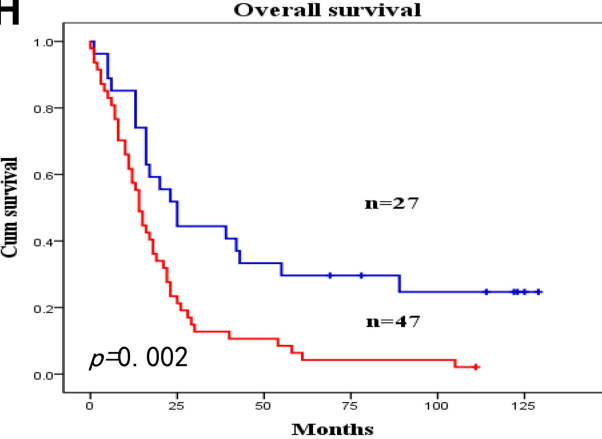

P53 Leve Thlow 十 Low-censored + High- censored

Figure 3 Kaplan-Meier survival analysis in stage I-II and III-IV patients with OSCC. Disease-free survival and overall survival curves for stage I-II OSCC patients with low and high expression levels of MDM2 (A and B) and p53 (C and D). Disease-free survival and overall survival curves for stage III-IV OSCC patients with low and high expression levels of MDM2 (E and $\mathbf{F})$ and p53 ( $\mathbf{G}$ and $\mathbf{H})$.

detection of 553 is recognized by most researchers as a common detection method, which reflects the potential TP53 mutation status of tumours. ${ }^{35,36}$ However, there is a difference between the overexpression of p53 and the mutation of TP53 in some tumours. Therefore, we did not detect the mutation status of p53 in the samples. On the other hand, due to the lack of fresh tissue specimens, we could not more accurately sequence the specimens. 

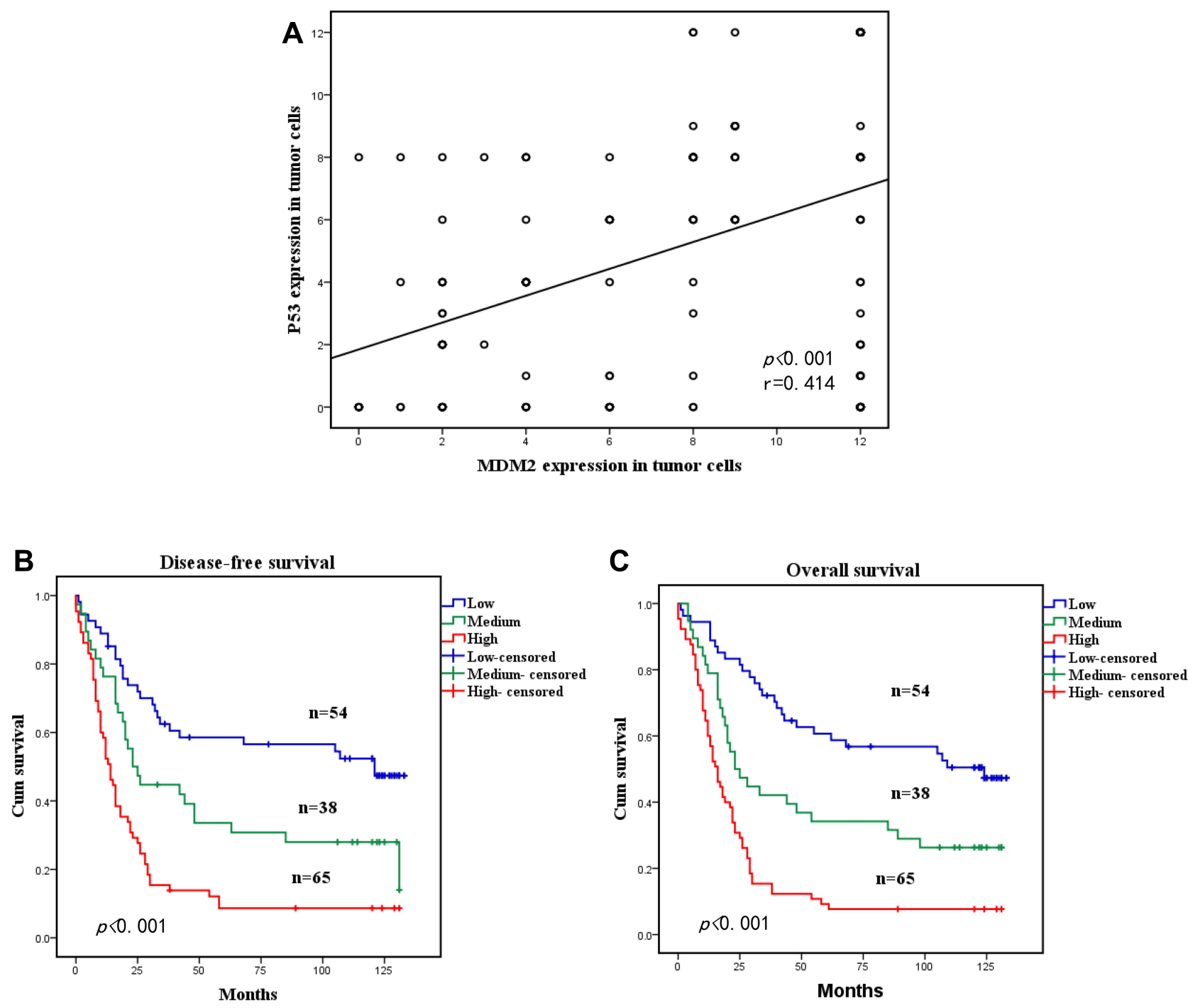

Figure 4 Correlation analysis between MDM2 and $\mathrm{p} 53$ expression in different cell populations and survival curves for OSCC patients according to their expression levels of MDM2 and p53 in tumour cells. (A) The expression levels of MDM2 and p53 in tumour cells were significantly positively correlated ( $p<0.001$, R=0.4I4). (B and C) Diseasefree survival and overall survival curves for patients according to the combined low, single high and combined high expression levels of MDM2 and $\mathrm{p} 53$ in tumour cells.

This study was a single-centre retrospective the role of these two genes in oesophageal cancer in cell study, and selection bias should be considered. The mechanism of action of p53 and MDM2 genes has not yet been explored. Therefore, we will further verify and animal models in future studies to verify our findings.

In conclusion, our research shows that the amplification rate of the high MDM2 expression group is higher

Table 5 Association Between MDM2 Expression and Amplification in Patients with Esophageal Squamous Cell Carcinoma

\begin{tabular}{|l|c|c|c|c|}
\hline \multirow{2}{*}{ Case } & MDM2 Amplification & \multicolumn{2}{|c|}{ MDM2 Expression } & \multirow{2}{*}{$p$ value } \\
\cline { 3 - 4 } & & Low & High \\
\cline { 3 - 4 } & & $(\mathrm{n}=\mathbf{6 2}$, Percent) & $(\mathrm{n}=\mathbf{9 5}$, Percent $)$ & \\
\hline 136 & No & $59(95.2)$ & $77(81.1)$ & $\mathbf{0 . 0 1 5}$ \\
21 & Yes & $3(4.8)$ & $18(18.9)$ & \\
\hline
\end{tabular}

Notes: Chi-square test was used in Table $5 ; p<0.05$ considered as statistically significant; bold values indicate statistically significant $(p<0.05)$. 
than that of the low expression group, and there is a strong correlation between MDM2 amplification and increased MDM2 expression. In addition, MDM2 and p53 can be used as independent risk factors for evaluating the prognosis of OSCC. Co-expression of the MDM2 protein and p53 represents a new prognostic hierarchical structure that can better assess the malignancy and prognosis of OSCC.

As a country with a very high incidence of OSCC, China urgently needs to achieve a higher early diagnosis rate of OSCC and effectively predict the disease progression and prognosis of patients with OSCC. Therefore, the discovery of more effective and convenient OSCC tumour markers has great clinical significance. In summary, our research indicates that MDM2 and p53, as important potential predictors of OSCC recurrence and prognosis, have important guiding significance for clinical diagnosis and treatment.

\section{Acknowledgments}

We thank the staff of the biochemical lab of Sun Yat-sen University Cancer Center who provided various biochemical markers and all the staff who supported our study.

\section{Author Contributions}

JY and LZ contributed equally. All authors made a significant contribution to the work reported, whether that is in the conception, study design, execution, acquisition of data, analysis and interpretation, or in all these areas; took part in drafting, revising or critically reviewing the article; gave final approval of the version to be published; have agreed on the journal to which the article has been submitted; and agree to be accountable for all aspects of the work.

\section{Funding}

This work was supported by the National Natural Science Foundation of China (Grant No.82003268, Grant No.81860441) and the Guangdong Province Natural Science Foundation (Grant No.2018A030310260).

\section{Disclosure}

The authors declare no conflicts of interest in this work.

\section{References}

1. Malhotra GK, Yanala U, Ravipati A, Follet M, Vijayakumar M, Are C. Global trends in esophageal cancer. J Surg Oncol. 2017;115 (5):564-579. doi:10.1002/jso.24592

2. Di Pardo BJ, Bronson NW, Diggs BS, Thomas CR, Hunter JG, Dolan JP. The global burden of esophageal cancer: a disability-adjusted life-year approach. World J Surg. 2016;40(2):395-401. doi:10.1007/s00268-015$3356-2$
3. Bray F, Ferlay J, Soerjomataram I, Siegel RL, Torre LA, Jemal A. Global cancer statistics 2018: GLOBOCAN estimates of incidence and mortality worldwide for 36 cancers in 185 countries. CA Cancer J Clin. 2018;68(6):394-424. doi:10.3322/caac.21492

4. Liang H, Fan JH, Qiao YL. Epidemiology, etiology, and prevention of esophageal squamous cell carcinoma in China. Cancer Biol Med. 2017;14(1):33-41. doi:10.20892/j.issn.2095-3941.2016.0093

5. Daly JM, Fry WA, Little AG, et al. Esophageal cancer: results of an American college of surgeons patient care evaluation study. $J \mathrm{Am}$ Coll Surg. 2000;190(5):562-572; discussion 572-563. doi:10.1016/ S1072-7515(00)00238-6

6. Abnet CC, Arnold M, Wei WQ. Epidemiology of esophageal squamous cell carcinoma. Gastroenterology. 2018;154(2):360-373. doi:10.1053/j.gastro.2017.08.023

7. Shan L, Yu J, He Z, et al. Defining relative mutational difficulty to understand cancer formation. Cell Discov. 2020;6:48. doi:10.1038/ s41421-020-0177-8

8. Yan W, Wistuba II, Emmert-Buck MR, Erickson HS. Squamous cell carcinoma - similarities and differences among anatomical sites. Am J Cancer Res. 2011;1(3):275-300. doi:10.1158/1538-7445.am2011-275

9. He LR, Liu MZ, Li BK, et al. High expression of EZH2 is associated with tumor aggressiveness and poor prognosis in patients with esophageal squamous cell carcinoma treated with definitive chemoradiotherapy. Int J Cancer. 2010;127(1):138-147. doi:10.1002/ijc.25031

10. Smyth EC, Lagergren J, Fitzgerald RC, et al. Oesophageal cancer. Nat Rev Dis Primers. 2017;3:17048.

11. Lane DP, Crawford LV. T antigen is bound to a host protein in SV40-transformed cells. Nature. 1979;278(5701):261-263. doi:10.10 38/278261a0

12. Shi L, Tian Q, Feng C, Zhang P, Zhao Y. The biological function and the regulatory roles of wild-type p53-induced phosphatase 1 in immune system. Int Rev Immunol. 2020;1-12.

13. Zhang Y, Qian M, Tang F, et al. Identification and analysis of p53-regulated enhancers in hepatic carcinoma. Front Bioeng Biotechnol. 2020;8:668. doi:10.3389/fbioe.2020.00668

14. Joerger AC, Fersht AR. The p53 pathway: origins, inactivation in cancer, and emerging therapeutic approaches. Annu Rev Biochem. 2016;85:375-404. doi:10.1146/annurev-biochem-060815-014710

15. Oren M, Levine AJ. Molecular cloning of a cDNA specific for the murine p53 cellular tumor antigen. Proc Natl Acad Sci U S A. 1983;80(1):56-59. doi:10.1073/pnas.80.1.56

16. Garty H, Klemperer G, Eisenbach M, Caplan SR. The direction of light-induced $\mathrm{pH}$ changes in purple membrane suspensions. Influence of $\mathrm{pH}$ and temperature. FEBS Lett. 1977;81(2):238-242. doi:10.1016/ 0014-5793(77)80526-7

17. Qin JJ, Li X, Hunt C, Wang W, Wang H, Zhang R. Natural products targeting the p53-MDM2 pathway and mutant p53: recent advances and implications in cancer medicine. Genes Dis. 2018;5(3):204-219. doi:10.1016/j.gendis.2018.07.002

18. Wang S, Zhao Y, Aguilar A, Bernard D, Yang CY. Targeting the MDM2-p53 protein-protein interaction for new cancer therapy: progress and challenges. Cold Spring Harb Perspect Med. 2017;7(5): a026245. doi:10.1101/cshperspect.a026245

19. Oliner JD, Saiki AY, Caenepeel S. The role of MDM2 amplification and overexpression in tumorigenesis. Cold Spring Harb Perspect Med. 2016;6(6):a026336. doi:10.1101/cshperspect.a026336

20. Chen Y, Wang DD, Wu YP, et al. MDM2 promotes epithelial-mesenchymal transition and metastasis of ovarian cancer SKOV3 cells. Br J Cancer. 2017;117(8):1192-1201. doi:10.1038/bjc. 2017.265

21. Wang BY, Cao J, Chen JW, Liu QY. Triptolide induces apoptosis of gastric cancer cells via inhibiting the overexpression of MDM2. Med Oncol. 2014;31(11):270. doi:10.1007/s12032-014-0270-7

22. Wu B, Chu X, Feng C, et al. Heat shock protein gp 96 decreases p53 stability by regulating Mdm2 E3 ligase activity in liver cancer. Cancer Lett. 2015;359(2):325-334. doi:10.1016/j.canlet.2015.01.034 
23. Sheng W, Dong M, Chen C, et al. Cooperation of Musashi-2, Numb, MDM2, and P53 in drug resistance and malignant biology of pancreatic cancer. FASEB J. 2017;31(6):2429-2438. doi:10.1096/fj.20 1601240R

24. Su HC, Wu SC, Yen LC, et al. Gene expression profiling identifies the role of Zac1 in cervical cancer metastasis. Sci Rep. 2020;10 (1):11837. doi:10.1038/s41598-020-68835-0

25. Zheng $\mathrm{H}$, Tie Y, Fang $\mathrm{Z}$, et al. Jumonji domain-containing 6 (JMJD6) identified as a potential therapeutic target in ovarian cancer. Signal Transduct Target Ther. 2019;4(1):24. doi:10.1038/ s41392-019-0055-8

26. Merten L, Agaimy A, Moskalev EA, et al. Inactivating mutations of RB1 and TP53 correlate with sarcomatous histomorphology and metastasis/recurrence in gastrointestinal stromal tumors. Am J Clin Pathol. 2016;146(6):718-726. doi:10.1093/ajcp/aqw193

27. Michalk M, Meinrath J, Kunstlinger H, et al. MDM2 gene amplification in esophageal carcinoma. Oncol Rep. 2016;35(4):2223-2227. doi:10.3892/or.2016.4578

28. Zhang YS, Shen Q, Li J. Traditional Chinese medicine targeting apoptotic mechanisms for esophageal cancer therapy. Acta Pharmacol Sin. 2016;37(3):295-302. doi:10.1038/aps.2015.116

29. Konopleva M, Martinelli G, Daver N, et al. MDM2 inhibition: an important step forward in cancer therapy. Leukemia. 2020;34 (11):2858-2874. doi:10.1038/s41375-020-0949-Z

30. Sawada R, Maehara R, Oshikiri T, et al. MDM2 copy number increase: a poor prognostic, molecular event in esophageal squamous cell carcinoma. Hum Pathol. 2019;89:1-9. doi:10.1016/j.humpath. 2019.04.002
31. Shimada H. p53 molecular approach to diagnosis and treatment of esophageal squamous cell carcinoma. Ann Gastroenterol Surg. 2018;2(4):266-273. doi:10.1002/ags3.12179

32. Sankalecha TH, Gupta SJ, Gaikwad NR, Shirole NU, Kothari HG. Yield of p53 expression in esophageal squamous cell cancer and its relationship with survival. Saudi J Gastroenterol. 2017;23(5): 281-286. doi:10.4103/sjg.SJG_56_17

33. Hong $\mathrm{Y}$, Miao X, Zhang X, et al. The role of P53 and MDM2 polymorphisms in the risk of esophageal squamous cell carcinoma. Cancer Res. 2005;65(20):9582-9587. doi:10.1158/0008-5472.CAN05-1460

34. Cheng TH, Hsu PK, Li AF, Hung IC, Huang MH, Hsu HS. Correlation of $\mathrm{p} 53, \mathrm{MDM} 2$ and $\mathrm{p} 14(\mathrm{ARF})$ protein expression in human esophageal squamous cell carcinoma. J Cancer Res Clin Oncol. 2009;135(11):1577-1582. doi:10.1007/s00432-009-0605-5

35. Köbel M, Ronnett BM, Singh N, Soslow RA, Gilks CB, McCluggage WG. Interpretation of P53 immunohistochemistry in endometrial carcinomas: toward increased reproducibility. Int J Gynecol Pathol. 2019;38(Suppl 1):S123-s131. doi:10.1097/ PGP.0000000000000488

36. Lee JM, Shun CT, Wu MT, et al. The associations of p53 overexpression with p53 codon 72 genetic polymorphism in esophageal cancer. Mutat Res. 2006;594(1-2):181-188. doi:10.1016/j.mrfmmm. 2005.09.003

\section{Publish your work in this journal}

Cancer Management and Research is an international, peer-reviewed open access journal focusing on cancer research and the optimal use of preventative and integrated treatment interventions to achieve improved outcomes, enhanced survival and quality of life for the cancer patient.
The manuscript management system is completely online and includes a very quick and fair peer-review system, which is all easy to use. Visit http://www.dovepress.com/testimonials.php to read real quotes from published authors. 